\title{
Determination of Intermolecular Potential Functions From Macroscopic Measurements*
}

\author{
Max Klein \\ Institute for Basic Standards, National Bureau of Standards, Washington, D.C. \\ (February 11, 1966)
}

\begin{abstract}
The second virial, adiabatic Joule-Thomson, viscosity, and diffusion coefficients predicted for a number of potentials are compared with those predicted for the $(12,6)$ potential. A quantitative picture, as a function of temperature, is obtained of the ability of each property to act as a probe of the potential function. The transport properties are found to be the most sensitive probes, the JouleThomson coefficient next, and the second virial coefficient least, the last property being essentially useless in the range $2.0<T^{*}<8.0$ on the $(12,6)$ reduced temperature scale.
\end{abstract}

Key Words: Potential function, second virial, viscosity, diffusion coefficient, Joule-Thomson, potential parameters.

\section{Introduction}

Statistical mechanics provides a molecular foundation for thermodynamics. This results in the expression of thermodynamic properties as functionals of the intermolecular potential functions of the constituent molecules. In principle, given the potential function appropriate to a given system one can calculate all of the thermodynamic properties of that system merely by turning the computational crank. In practice, matters are not so simple both because the relationships to be evaluated are enormously complicated and because the potential functions are not known with sufficient accuracy.

The most accurate of the statistical mechanical expressions contain $N$-body potential functions $\left(N \sim 10^{23}\right)$ which are impossibly difficult to calculate. Simplifying assumptions can be made which often, as in the virial expansion, result in a power series in some parameter (e.g., the density) whose coefficients depend on lower order $N$-body potentials $(N=2$, 3, . . .). Since even the three-body potential is exceedingly difficult to calculate, the assumption of pairwise potential additivity must generally be included. With this assumption, the thermodynamic properties, in the statistical mechanical expressions, can be made to depend on only the pair potential function. Most theories for the further simplification of the complicated expressions proceed from this point on the assumption that the pair potential function is known. These theories result in simpler, but generally still complicated, relationships between the thermody-

* Supported, in part, by the Air Force Systems Command, Arnold Engineering Development Center, Tullahoma, Tenn., Delivery Order Number (40-600) 66-22 Program Element 61445014, AF Project 8951. namic properties and the potential function. The evaluation of such theories can be a formidable task. All too often their final evaluation is obscured $[1]^{1}$ considerably by the fact that pair potential functions are, in practice, only imperfectly known.

The intermolecular potential function together with a sufficiently accurate theory can be used to extrapolate far beyond the bounds of available experimental data [2], something which is not possible using completely empirical methods. Such extrapolations are very strongly dependent on the potential function and can be considerably in error when the wrong potential function is used.

Clearly the determination of accurate intermolecular potential functions is of some importance. In this work we have sought to understand some of the methods generally used to determine such functions, particularly with respect to the question of the uniqueness of the potentials obtained. A lack of uniqueness exists when a set of experimental data for a given property can be correlated equally well using the appropriate theory and any of two or more potential functions. Where a lack of uniqueness exists, it becomes necessary to attempt to determine if there is a lack of sensitivity inherent in the theoretical quantity itself or if it is lack of experimental precision which makes it possible to fit the data equally well with two or more theoretical curves. These are equivalent to the following two questions. First, how well can the property of interest, in principle, distinguish among potential functions and, second, how well can it distinguish among such functions at the present time, given present day experimental precision. The answer to the first question is a perma-

Figures in brackets indicate the literature references at the end of this paper. 
nent one while the answer to the second one changes as experimental technique is refined, approaching the first answer in the limit of zero experimental error. We have restricted ourselves to the first question and discuss the second one only in passing mainly to place our results in a practical light. The answer to the first question is of considerable practical importance since it points out where refined experimental techniques will not produce more information about the potential function.

In principle the pair potential functions can be calculated in an a priori fashion using quantum mechanics by calculating the potential energy of two molecules as a function of nuclear spearation. Since one needs to consider all the electrons in each molecule, this is also an intractable $N$-body problem, $N$ now being the total number of electrons involved. This leaves one no alternative but to turn the problem around and determine the potential, in some manner, from experiment. In practice, the procedure is reduced to a semiempirical one. A functional form is assumed for the potential whose choice is based, in part, on theoretical arguments. In this form are included parameters whose values (and hence the detailed potential) are to be determined from experiment. The parameter determination is made by substituting the potential into statistical mechanical expressions for some macroscopic property and comparing the result with experiment [3]. Best results are to be expected when the theory is one whose dependence on the pair potential is strongly based as is the case, for example, in the low density limit of certain theories.

In this paper we shall discuss the use of the zerodensity viscosity, diffusion, and adiabatic JouleThomson coefficients and the second virial coefficient as ways of obtaining the potential parameters. For each of these, the pair potential appears in the integrand of an expression for the macroscopic property. This suggests that, given enough experimental data of sufficient accuracy, one might be able to invert the theoretical expressions and determine the potential as a unique functional of the experimental data. For the second virial coefficient, however, Le Fevre and Keller and Zumino [4] have shown that the potential is not determined uniquely by the data, even in principle. In this work we demonstrate this lack of uniqueness quantitatively for particular potentials. We show there exists a lack of uniqueness for each of the macroscopic properties considered, although it is somewhat less pronounced for the transport properties than for the equilibrium properties. For each property, the lack of uniqueness is found to be more pronounced in one temperature range than in another. Strong positive statements can then be made about the temperatures at which experiments designed largely to determine potential functions should not be performed for particular substances. We are also able to show the simultaneous fit of certain of these properties to be sensitive to differences in the potential function.

\section{Computational Method}

Because of its relative simplicity, we shall use the second virial coefficient to illustrate the details of the computation. The other properties are handled in essentially the same manner. We shall restrict the discussion to two parameter potentials. This is no real restriction since a three-parameter potential can be treated as a family of two parameter potentials one for each value of the third parameter.

The second virial coefficient is related to the potential function, $\varphi$, by the relation [3]

$$
B(T)=b_{0} B^{*}\left(T^{*}\right)=-b_{0} \int_{0}^{\infty}\left[\exp \left(-\frac{\varphi^{*}\left(r^{*}\right)}{T^{*}}\right)-1\right] r^{* 2} d r^{*}
$$

where $b_{0}=\frac{2 \pi N \sigma^{3}}{3}, T^{*}=k T / \epsilon, \varphi^{*}=\varphi / \epsilon$, and $r^{*}=r / \sigma$. Here, as usual, $\sigma$ is a characteristic length related to $\varphi$ and $\epsilon$ is the depth of the potential well. $N$ is Avogadro's number. For a given potential, a given $T$, and in the left-hand side, a given experimental value of $B$ at that $T$, (1) contains only the two unknowns $\sigma$ and $\epsilon$. In this work, in place of experimental $B(T)$ values, we supply to the left-hand member of (1) the second virial coefficient for a potential function other than the one appearing in the right-hand side. Thus, if the subscripts 1 and 2 refer to the two potentials, (1) becomes

$$
B(T)=\left(b_{0}\right)_{1} B_{1}^{*}\left(T_{1}^{*}\right)=\left(b_{0}\right)_{2} B_{2}^{*}\left(T_{2}^{*}\right)
$$

or

$$
B_{1}^{*}\left(T_{1}^{*}\right)=\frac{\left(b_{0}\right)_{2}}{\left(b_{0}\right)_{1}} \int_{0}^{\infty}\left\{\exp \left(-\frac{\varphi_{2}^{*}\left(r^{*}\right)}{T_{2}^{*}}\right)-1\right\} r^{* 2} d r^{*}
$$

where $B_{1}^{*}\left(T_{1}^{*}\right)$ is a functional of $\varphi_{1}^{*}\left(r^{*}\right)$. Now $T_{1}^{*}=k T / \epsilon_{1}$ and $T_{2}^{*}=k T / \epsilon_{2}$ hence, for a given $T, T_{2}^{*}=\frac{\epsilon_{1}}{\epsilon_{2}} T_{1}^{*}$ so that

$$
B_{1}^{*}\left(T_{1}^{*}\right)=\frac{\left(b_{0}\right)_{2}}{\left(b_{0}\right)_{1}} \int^{\infty}\left\{\exp \left(-\frac{\varphi_{2}^{*}\left(r^{*}\right) \epsilon_{2}}{T_{1}^{*} \epsilon_{1}}\right)-1\right\} r^{* 2} d r^{*} .
$$

This is an equation containing two unknowns, namely, the ratios $\epsilon_{2} / \epsilon_{1}$ and $\left(b_{0}\right)_{2} /\left(b_{0}\right)_{1}$. These ratios are completely determined, given another equation connecting some other functional of the potential for the two potentials. We have taken, for this second functional, the first derivative of $B$ with respect to the logarithm of $T$ [5]. We thus determine, at each temperature, that pair of ratios, $\epsilon_{2} / \epsilon_{1}$ and $\left(b_{0}\right)_{2} /\left(b_{0}\right)_{1}$ which results in the equality, for the two potentials, of both the second virial coefficient and its first derivative with respect to the logarithm of the temperature. Ordinarily, the ratios are different at different temperatures, hence we shall actually determine their temperature depend- 
ence. By dividing the equation which represents the requirement of equality of the first derivatives by that representing the equality of the second virial coefficients themselves, one obtains a single equation for the equality of the logarithmic derivatives. The working equation becomes

$$
\frac{d \ln B_{1}^{*}\left(T_{1}^{*}\right)}{d \ln T_{1}^{*}}=\frac{d \ln B_{2}^{*}\left(T_{2}^{*}\right)}{d \ln T_{2}^{*}} .
$$

This is a single implicit equation in the ratio $\epsilon_{2} / \epsilon_{1}$, the dependence on $\left(b_{0}\right)_{2} /\left(b_{0}\right)_{1}$ being removed in the process of differentiating the logarithm.

On the substitution of the set of values $B_{1}^{*}\left(T_{1}^{*}\right)$ in the left-hand member, (2) becomes a family of curves for the ratio $\epsilon_{2} / \epsilon_{1}$, as a function of the ratio $\left(b_{0}\right)_{2} /\left(b_{0}\right)_{1}$, one curve for each value of $T_{1}^{*}$ [7]. These can also be used for obtaining information about the potential function [8], although the results so obtained cannot be easily presented in a manner suitable for our purposes. We have therefore used a different approach. We have computed, for each of a number of potentials, $B^{*}$ and $B^{* *}=T^{*}\left(\frac{d B^{*}}{d T^{*}}\right)$ and from these

$$
S=\frac{T^{*}}{B^{*}} \frac{d B^{*}}{d T^{*}}=\frac{B^{* *}}{B^{*}}=\frac{d \ln B^{*}}{d \ln T^{*}} .
$$

These are used to solve (3) in the following way. Each value of $T_{2}^{*}$ has associated with it a value $S_{2}$. Using inverse interpolation, the value of $T_{1}^{*}$ is found for which $S_{1}=S_{2}$. The ratios $\epsilon_{2} / \epsilon_{1}$ and $\left(b_{0}\right)_{2} /\left(b_{0}\right)_{1}$ are then computed simply from

$$
\frac{\epsilon_{2}}{\epsilon_{1}}=\frac{T_{1}^{*}}{T_{2}^{*}} \text { and } \quad \frac{\left(b_{0}\right)_{2}}{\left(b_{0}\right)_{1}}=\frac{B_{2}^{*}\left(T_{2}^{*}\right)}{B_{1}^{*}\left(T_{1}^{*}\right)} .
$$

This procedure can be illustrated graphically with the help of figures 1 and 2 . These contain plots of $S$ for the second virial coefficient [9] versus the logarithm of the reduced temperature for several representative potential functions. Temperatures below the Boyle temperature appear in figure 1, those above that temperature in figure 2 . Note that the $S$ values for each potential are plotted against the logarithm of the reduced temperature for that potential. Equation (3) is solved graphically at each $T_{1}^{*}$ by measuring the horizontal distance between the ordinate associated with $T_{1}^{*}$ on curve 1 and the same ordinate on curve 2 . This distance is then just

$$
\log T_{2}^{*}-\log T_{1}^{*}=\log \left(T_{2}^{*} / T_{1}^{*}\right)=\log \left(\epsilon_{1} / \epsilon_{2}\right)
$$

It should be noted that where there are values of $S_{2}$ greater than the maximum $S_{1}$ value, solution of (3) is impossible. This occurs (see fig. 1) for the $(9,6)$ potential compared to the $(12,6)$.

Note that both the value and slope of $B_{1}^{*}$ are fit by $B_{2}^{*}$ at $T_{1}^{*}$. It follows, therefore, that $B_{2}^{*}$ will actually produce a relatively good fit to $B_{1}^{*}$ in a small neighborhood

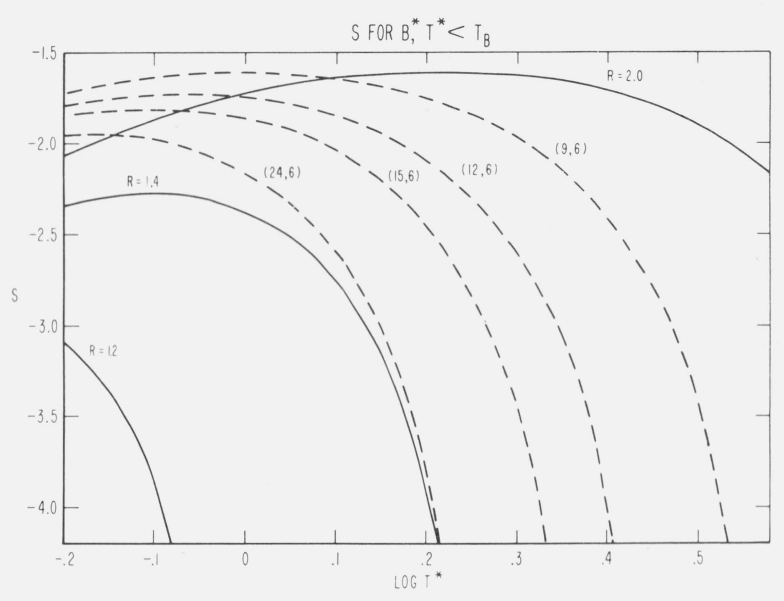

Figure 1. S for the second virial coefficient of several potentials, $\mathrm{T}^{*}$ less than the Boyle temperature.

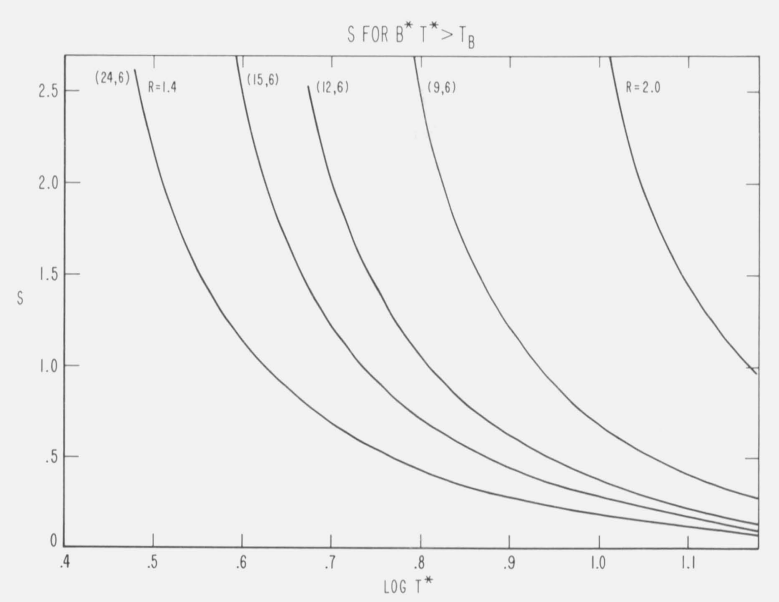

Figure 2. S for the second virial coefficient of several potentials, $\mathrm{T}^{*}$ greater than the Boyle temperature.

around $T_{1}^{*}$ for the parameter ratio obtained by solving (3) at $T_{1}^{*}$. Furthermore, when this ratio is found to be independent of $T_{1}^{*}$ over a range of values of $T_{1}^{*}, B_{2}^{*}$ provides an excellent fit to $B_{1}^{*}$ over that range.

In this calculation, the Boyle temperature serves as a natural dividing point between two temperature ranges. Since $B$ is zero at that temperature, the derivatives in (3) are not defined. Furthermore, since $B$ changes sign while $\frac{d B}{d T}$ does not, $S$ also changes sign.

Hence, values of $S$ taken from temperatures above the Boyle temperature for one potential cannot possibly be made to fit those taken from below it for the other potential. Therefore, we have treated these two ranges separately coming as close to the Boyle temperature as desired from either side.

As mentioned in the introduction, the zero density adiabatic Joule-Thomson, viscosity, and diffusion coefficients are also considered here. These are also treated as outlined above. The equation (3) for each 
of these is replaced in turn by the requirement that the quantities $\frac{d \ln \left[\mu C_{P}^{(0)}\right]}{d \ln T^{*}}$ for the adiabatic Joule-Thomson coefficient, $\frac{d \ln \eta T^{*-1 / 2}}{d \ln T^{*}}$ for the viscosity, and $\frac{d \ln \left[\mathscr{D} T^{*}{ }^{-3 / 2}\right]}{d \ln T^{*}}$ for the diffusion coefficient be equal for the two potentials. Now [3] $\mu^{*} C_{P}^{(0)}=T^{*} \frac{d B^{*}}{d T^{*}}-B^{*}$ $=B^{\prime *}-B^{*}$ so that the connection between this quantity and the potential function is essentially the same as between the second virial coefficient and the potential. From this last,

$$
T^{*} \frac{d}{d T^{*}}\left[\mu C_{P}^{(0)}\right]=B^{\prime *} \quad \text { and } \frac{d \ln \left[\mu C_{P}^{(0)}\right]}{d \ln T^{*}}=\frac{B^{\prime *}}{B^{1 *}-B^{*}}
$$

where $B^{\prime \prime *}=T^{* 2} \frac{d^{2} B^{*}}{d T^{* 2}}$. Here, as with the second virial coefficient, there is a temperature which divides the calculation into two parts. Now, however, the zero density inversion temperature (defined by $B^{\prime *}=B^{*}$ ) plays the role previously played by the Boyle temperature.

The connection between the viscosity and diffusion coefficients and the intermolecular potential function is contained in the so-called collision integrals [3]

$$
\begin{aligned}
\Omega^{(l, s) *}\left(T^{*}\right) & \frac{2}{(S+1) ! T^{*(s+2)}} \\
& \int_{0}^{\infty} e^{-g^{* 2 / T *} g^{*(2 s+3)}} Q^{(l) *}\left(g^{*}\right) d g^{*}
\end{aligned}
$$

where

$$
Q^{(l) *}\left(g^{*}\right)=\frac{2}{1-\frac{1}{2} \frac{1+(-1)}{1+l}} l \int_{0}^{\infty}\left(1-\cos ^{l} \chi\right) b^{*} d b^{*}
$$

with the intermolecular potential function being contained in the equation for the scattering angle

$$
\chi\left(g^{*}, b^{*}\right)=\pi-2 b^{*} \int_{r *}^{\infty} \frac{d r^{*} / r^{*}}{\sqrt{1-b^{* 2} / r^{* 2}-\phi\left(r^{*}\right) / g^{* 2}}}
$$

$r_{m}^{*}$ being the distance between a pair of molecules at the time of closest approach.

In terms of these, the zero density viscosity and diffusion coefficients can be written [3]

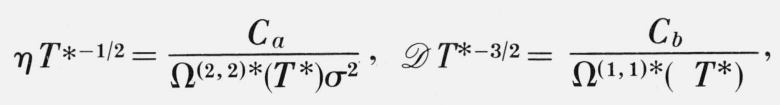

where $C_{a}$ and $C_{b}$ are constants whose precise values are of no particular interest here. It can be easily shown that

$$
\frac{d \ln \Omega^{(l, j) *}}{d \ln T^{*}}=(j+2) \frac{\Omega^{(l, j+1) *}}{\Omega^{(l, j) *}}-(j+2) .
$$

From this it follows that, for the viscosity, eq (3) is replaced by the requirement that $4 \frac{\Omega^{(2,3) *}}{\Omega^{(2,2) *}}-4$ be equal for the two potentials. For the diffusion coefficient, on the other hand, the quantity $3 \frac{\Omega^{(1,2) *}}{\Omega^{(1,1) *}}-3$ must be equal for the potentials. Since $\eta$ and $\mathscr{D}$ are each nonzero, there is no dividing temperature analogous to the Boyle temperature for these properties. The $S$ values corresponding to these properties are plotted in figures 3 and 4.

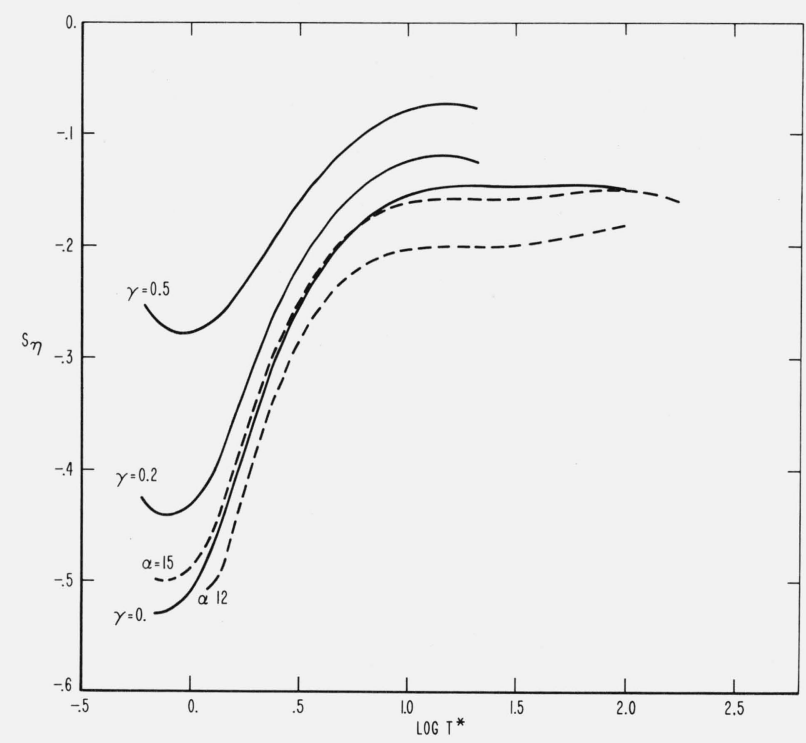

FigurE 3. S for the viscosity coefficient of several potentials.

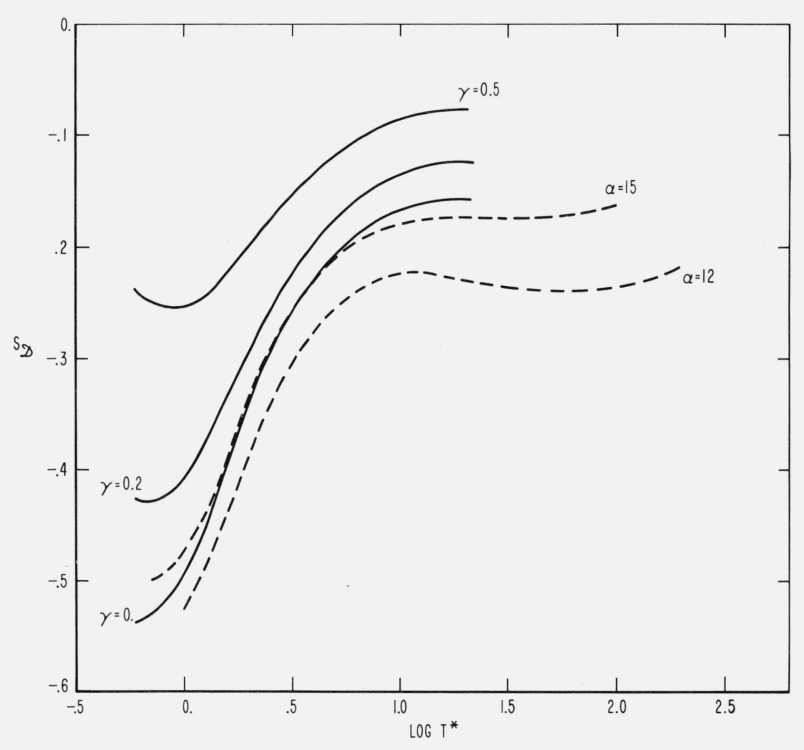

Figure 4. S for the diffusion coefficient of several potentials. 
The macroscopic properties of most experimental systems have been correlated using the $(12,6)$ potential function [10]. As a result $\epsilon / k$ values for that potential can be found in the literature for just about all possible systems of interest. Furthermore, it has been shown that, for very many substances, a very simple relationship exists between the $\epsilon / k$ value for the $(12,6)$ potential and the temperatures for the critical and normal boiling points for that substance $[3,10,11]$. As a result, good first guesses for the $\epsilon / k$ values for the $(12,6)$ potential can be made for any system for which either the critical temperature or normal boiling point is known. For these reasons we have chosen to compare each potential with the $(12,6)$ function using the reduced temperature for the latter as the reference temperature $T_{1}^{*}$. The conversion to real experimental temperatures for any system merely requires multiplication by the (generally available) $\epsilon / k$ value of the $(12,6)$ potential for that system.

\section{Results}

The Second Virial Coefficient. Our results for the second virial coefficient are given in figures 5 to 8 as plots of the ratios $\epsilon_{2} / \epsilon_{1}$ versus the Lennard-Jones $(12,6)$ reduced temperature. Figure 11 contains plots of the ratio $\left(b_{0}\right)_{2} /\left(b_{0}\right)_{1}$. The potential functions considered are the following:

The $(m, n)$ :

$$
\phi^{*}\left(r^{*}\right)=\frac{\phi(r)}{\epsilon}=\left\{\frac{1}{\left(\frac{n}{m}\right)^{\frac{m}{m-n}}-\left(\frac{n}{m}\right)^{\frac{n}{m-n}}}\right\}\left[\left(\frac{1}{r^{*}}\right)^{m}-\left(\frac{1}{r^{*}}\right)^{n}\right]
$$

where $r^{*}=r / \sigma, \sigma$ is that value of $r$ for which $\varphi^{*}=0$. The Kihara:

$$
\begin{aligned}
& \phi^{*}\left(r^{*}\right)=\infty, r^{*} \leqslant \gamma^{*} \\
& \phi^{*}\left(r^{*}\right)=4\left[\left(\frac{1}{r^{*}-\gamma^{*}}\right)^{12}-\left(\frac{1}{r^{*}-\gamma^{*}}\right)^{6}\right], r^{*}>\gamma^{*}
\end{aligned}
$$

where $r^{*}=\frac{r}{\sigma-2 a}$ and $\gamma^{*}=\frac{2 a}{\sigma-2 a}, a$ being the core
radius. The Exp-6:

$$
\phi^{*}\left(r^{*}\right)=\frac{1}{1-\frac{6}{\alpha}}\left[\frac{6}{\alpha} \exp \left(\alpha\left(1-r^{*}\right)\right)-\left(\frac{1}{r^{*}}\right)^{6}\right]
$$

where $r^{*}=r / r_{m}, r_{m}$ being that value of $r$ for which $\varphi^{*}=-1$.
The Square Well:

$$
\begin{aligned}
& \varphi^{*}\left(r^{*}\right)=\infty, r^{*} \leqslant 1 \\
& \varphi^{*}\left(r^{*}\right)=-1,1 \leqslant r^{*} \leqslant R^{*} \\
& \varphi^{*}\left(r^{*}\right)=0, r^{*} \geqslant R^{*}
\end{aligned}
$$

where $r^{*}=r / \sigma$.

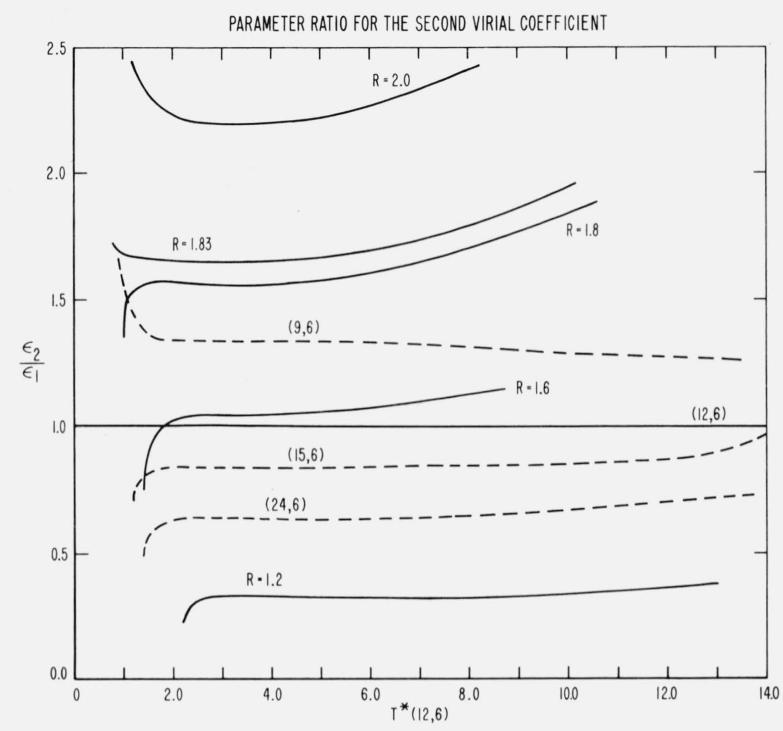

FIGURE 5. The parameter ratios $\epsilon_{2} / \epsilon_{1}$ for the second virial coefficients of the $(\mathrm{m}, 6)$ and square well potentials with respect to the $(12,6)$ potential.

Note particularly the flatness of the curves for the $(m, 6)$ potentials.

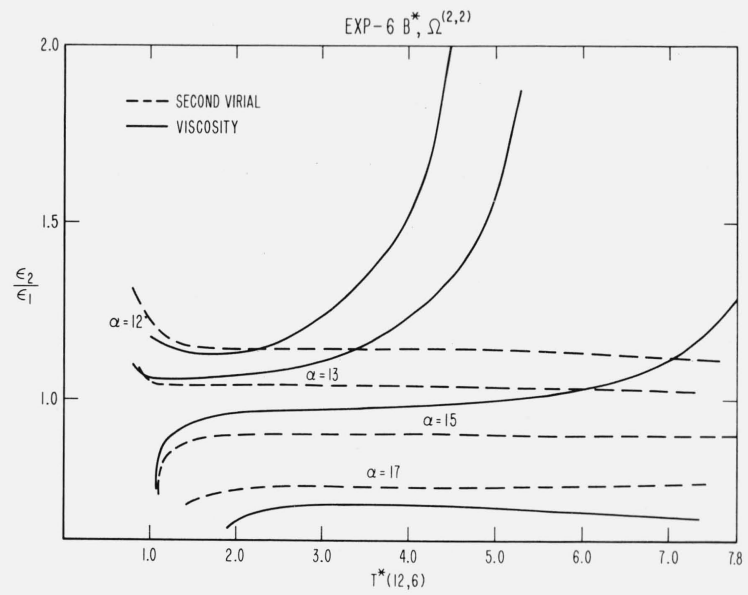

FIGURE 6. The parameter ratios $\epsilon_{2} / \epsilon_{1}$ for both the second virial and viscosity coefficients of the exp-6 potential with respect to the $(12,6)$ potential.

Note that the former are flat while the latter are not flat. 


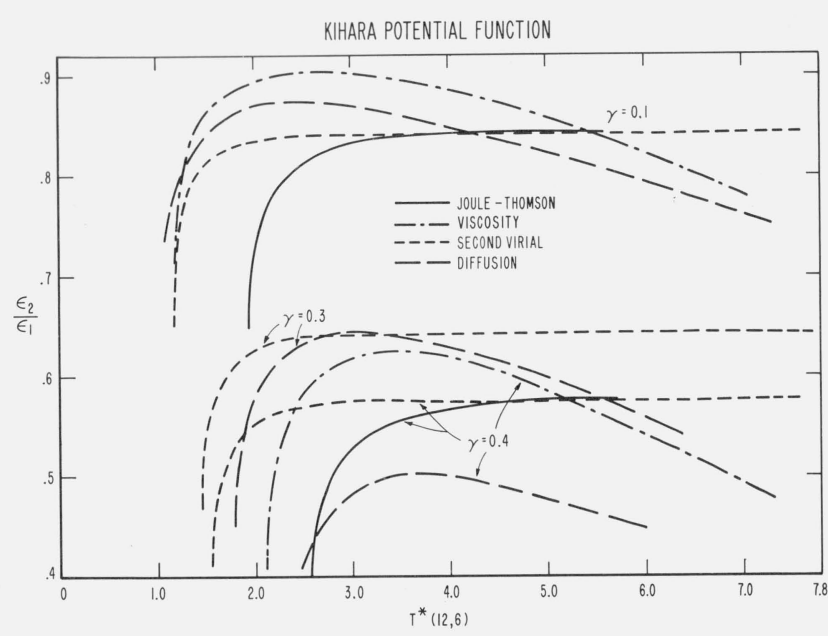

FIGURE 7. The parameter ratios $\epsilon_{2} / \epsilon_{1}$ for all four properties for the Kihara potential with respect to the $(12,6)$ potential.

Note the absence of any single ratio for which both the equilibrium and transport properties are flat.

Figure 5 contains results for the $(m, 6)$ and square well potentials, while results for the exp-6 and Kihara potentials are contained in figures 6 and 7 . Results for the $(12, n)$ appear in figure 8 . Each curve represents the comparison between a potential and the $(12,6)$ potential, the subscript 2 always referring to the former potential. For each curve there is a smooth transition through the Boyle temperature indicating that the singularity in $S$ at that temperature presents no problem. There exists, for each potential, a temperature range around the Boyle point such that in that range $\epsilon_{2} / \epsilon_{1}$ is essentially independent of $T^{*}$. This means that, for temperatures in that range, the $(12,6)$ and the potential with which it is compared are equivalent [12]. In fact, each curve is flat to such an extent in this range that a choice cannot be made between the $(12,6)$ potential and the one with which it is compared based on experimental second virial coefficient data taken entirely within the range, even when these data are obtained with an impossibly high precision. What is particularly striking is that there is a single temperature range in which all the curves are flat. This range becomes exceedingly large if one does not include the square well potential in the comparisons. Obviously, there is a reduced temperature regime in which the second virial coefficient is particularly useless as a probe of the potential function. What these results show specifically is that the second virial coefficient cannot be used in this range to distinguish among any of the members of the $(m, 6)$, exp-6, Kihara, $(12, n)$ and square well families of potentials. The list would presumably have been broadened had we considered other classes of functions.

Of considerable interest are the results obtained when the $(12, n)$ potentials are compared with the $(12,6)$. The curves obtained for these are essentially the same as those for the $(m, 6)$ emphasizing the fact that the second virial coefficient cannot be used to determine the exponent of the attractive part of the potential. The requirement that the attractive exponent be 6 is, rather, a restriction placed on the potential based on a priori information, at least for the second virial coefficient. Thus, we see that the second virial coefficient is determined by the general shape of the potential and not necessarily by its details. This has previously been demonstrated formally by Le Fevre [4] and by Keller and Zumino [4]. They showed

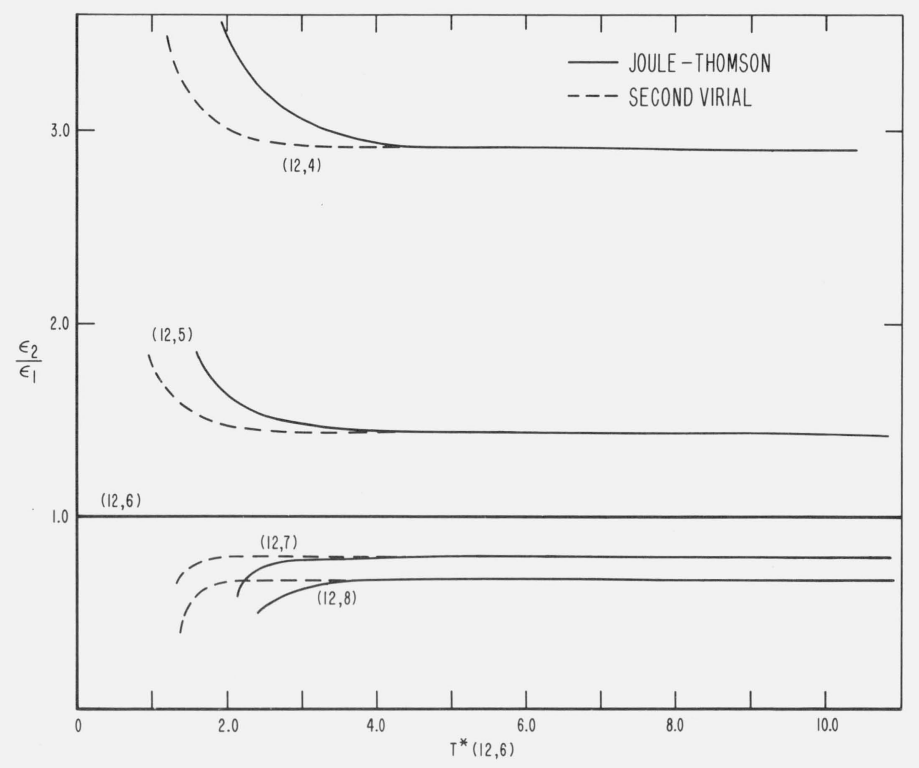

FIGURE 8. The parameter ratios $\epsilon_{2} / \epsilon_{1}$ for both the second virial and Joule-Thomson coefficients for the $(12, \mathrm{n})$ potential with respect to the $(12,6)$ potential.

Note that for temperatures above the Boyle point all curves are flat making it impossible to select a value of $n$ at such temperatures. 
that all potentials for which the sum of a certain pair of integrals, one over the repulsive part and one over the attractive part, were equal yielded the same second virial coefficient. A special case of their result is that all potentials with the same repulsive part and whose attractive parts have the same width as a function of depth (i.e., but whose bowls are possibly displaced laterally) yield the same second virial coefficient.

At temperatures outside the flat portion, the ratio $\epsilon_{2} / \epsilon_{1}$ is no longer constant. Below $T^{*}=2.0$ a particularly rapid variation is indicated. The origin of this rapid variation can be seen in figure 1 to be due to large differences in the slopes of the corresponding $S$ curves for equal values of the ordinate. According to figure 1 , there are also potentials for which there is a temperature range in which there are $S$ values larger than the maximum $S$ value of the $(12,6)$ potential. The $(9,6)$ function is an example. In such cases, solution of (3) is impossible. In other words, no ratio $\epsilon_{2} / \epsilon_{1}$ exists by means of which one can obtain simultaneous equality of both $B$ and $T \frac{d B}{d T}$ for the two potentials.

The existence of a rapid variation of $\epsilon_{2} / \epsilon_{1}$ with $T^{*}$ at low temperatures would seem to indicate a very strong sensitivity, at such temperatures, to differences in the potential functions. Inability to solve (3) indicates an even stronger sensitivity to such differences. However, the sensitivity indicated applies strictly to exact data and the exact simultaneous fit of $B$ and $T \frac{d B}{d T}$. As the requirement on the exactness of the fit is relaxed, the sharpness of the variation of $\epsilon_{2} / \epsilon_{1}$ with $T^{*}$ is reduced. The introduction of these uncertainties in effect replaces each $S$ curve of figure 1 by an area bounded by two $S$ curves. One has then to compare two broadly defined $S$ areas rather than two sharply defined $S$ curves. This can make an overlap of ordinates possible near the maximum of the $(12,6)$ curve and hence make solution of $(3)$ possible where it previously was not. Furthermore, in comparing the two $S$ areas one has the possibility of choosing the two $S$ curves, one within each area, whose slopes are most nearly alike. This could result in a reduction in the rapid variation of $\epsilon_{2} / \epsilon_{1}$ with $T^{*}$ at low temperatures. For application to inherently imprecise experimental data, the low temperature region therefore becomes a much less sensitive probe of the potential than is indicated in figure 5. That is, an approximate fit to an accuracy compatible with experimental error might be possible where an exact fit, as indicated by figures 1 and 5, is impossible or, at best, difficult.

There is another, more fundamental reason why the rapid variation of $\epsilon_{2} / \epsilon_{1}$ with $T^{*}$ at low temperatures does not necessarily mean a sensitivity to differences in the potential functions. In this calculation, we have required the equality of both the second virial coefficient and its slope for the two potentials. This applies a much more stringent condition on the functions than is required in the correlation of experi- mental data. In the latter case, it is asked only that the theoretical values of $B(T)$ come as close as possible to the experimental ones. Nothing is asked of the slope of the second virial coefficient. Clearly two functions may each fit the data within experimental precision, yet their slopes may disagree by considerably more than the precision of the present calculation.

On the other hand, the fact that we place such strong conditions on the potential enables us to make strong statements where the second virials and their first derivatives for the potentials are essentially indistinguishable from each other. Obviously where our calculations cannot distinguish between potentials, a correlation which makes use of experimental data will be able to distinguish between them to a much lesser extent. Clearly, therefore, experiments designed to measure the second virial coefficient for purposes of learning something about the potential function should never be carried out above $T^{*}=2.0$ on the $(12,6)$ scale. In fact, existing data in that range should not be included in a determination of potential parameters since such data will supply experimental error without supplying any discrimination and so will reduce, for example, the ratios of the standard deviations obtained from fits of different potentials. This reduced temperature is easily converted to real temperatures for a particular substance given the $\epsilon / k$ value for the $(12,6)$ potential for that substance. For example, for argon, the data must have been taken at $T<240{ }^{\circ} \mathrm{K}$ while for xenon, the corresponding requirement is $T<450^{\circ} \mathrm{K}$.

It is clear from figures 5 and 6 that in each class (i.e., square well, exp-6, etc.) there exists a potential for which the ratio $\epsilon_{2} / \epsilon_{1}$ is essentially independent of $T^{*}$ even at low temperatures. For the exp-6 this occurs for $\alpha$ slightly larger than 13. For the square well, it occurs for $R^{*}$ approximately equal to 1.82 . One expects this also to be true for other classes of three parameter potential classes of which the $(12,6)$ is not a member. That is, there will exist a member of each such class which is equivalent to the $(12,6)$ in predicting the second virial coefficient over a large temperature range including low temperatures. Since the $(12,6)$ potential function was chosen as the reference potential in an entirely arbitrary fashion, there is no need to restrict this result to it. Thus, one can actually state that given any potential function, it is possible to find in every three parameter family of functions of which it is not a member, a potential function with which one can obtain a classical second virial coefficient whose value and slope differ from those calculated with the given potential by an amount much less than the best available experimental precision over a temperature range starting at extremely low temperatures and extending to temperatures well above experimental conditions for almost all substances. In short, the second virial coefficient is seen to be at best a three parameter quantity with regard to the potential function and any attempt to use functions with more parameters necessarily leads 
to redundancies. This is presumably what is behind the inability to obtain unique parameters in recent attempts to determine the potential function from second virial coefficient data using many parameter potential functions.

As expected, deviations from this can occur at high temperatures. That is, where the repulsive parts of the potentials differ sufficiently in character, the high temperature region can be used to choose among different classes. Thus a choice can be made between the extremely different $(12,6)$ and square well potentials if the data covers a range above $T^{*}=7.0$ on the $(12,6)$ temperature scale. A choice between the somewhat less different exp-6 and $(12,6)$ potentials, on the other hand, requires data at $T^{*}>10.0$ on the same scale. These are very high temperatures for most substances. For argon this latter requirement is $T>1200{ }^{\circ} \mathrm{K}$ while for xenon it is $T>2250{ }^{\circ} \mathrm{K}$.

The ratio $\left(b_{0}\right)_{2} /\left(b_{0}\right)_{1}$ for the second virial coefficient behaves in essentially the same way as does $\epsilon_{2} / \epsilon_{1}$. There is, therefore, no need to discuss its behavior separately.

Attempts to select, from several functions, a potential function for a particular system have sometimes been based on fits to second virial coefficient data which lie almost entirely within the flat portions of figures 5 to 8 . Examples are the correlations of Whalley and Schneider [13] and of Mason and Rice [14]. In table 1 we have reproduced the standard deviations obtained by Whalley and Schneider for several potential functions. Note that only in the case of xenon, where half of the points lie outside the flat portions of figures 5 to 8 , is there a strong discrimination among the potential functions. For the krypton data, one certainly has no basis for the selection of one potential over another while for argon the choice is, at best, a marginal one.

TABLE 1. Standard deviations obtained by Whalley and Schneider ${ }^{\text {a }}$

\begin{tabular}{l|c|c|c|c|c|c|c|c}
\hline \hline & $(9,6)$ & $(12,6)$ & $\alpha=12$ & $\alpha=13$ & $\alpha=14$ & $\alpha=15$ & $\begin{array}{c}\text { Total } \\
\text { number of } \\
\text { isotherms }\end{array}$ & $\begin{array}{c}\text { Number of } \\
\text { isotherms } \\
T^{*}<2.0 \\
(12,6)\end{array}$ \\
\cline { 2 - 7 } & & & & & & & \\
Argon & 0.39 & 0.42 & & & & 0.53 & 15 & 2 \\
Krypton & .35 & .36 & 0.46 & 0.47 & 0.41 & .42 & 9 & 2 \\
& 1.20 & .82 & .88 & & .70 & .62 & 12 & 6 \\
\hline
\end{tabular}

${ }^{a}$ E. Whalley and W. G. Schneider, J. Chem. Phys. 23, 1644 (1955).

From our results one can also see the futility of basing the choice of a potential function on the basis of the best fit of experimental data to a single two parameter function. To demonstrate this, let us take as an experimental system that system whose intermolecular potential function is exactly the $(12,6)$ function. Figure 6 then represents an attempt to fit the "experimental" second virial coefficients to those predicted for the exp-6 potential. The best fit is obtained for that potential which gives the most nearly flat curve in figure 6 . According to that figure, this best fit occurs for a value of $\alpha$ slightly greater than 13. The potential function defined by that value of $\alpha$ and the pair of parameters which give this best fit can then be associated with our "experimental" system and possibly used as such in other theories. Suppose now that instead of doing fits for a series of values of $\alpha$ we had just done the fit for a single value of $\alpha$. Clearly, for every value of $\alpha$, a pair of parameters exists which gives the best fit to the "experimental" data for that value of $\alpha$. However, the potential represented by that value of $\alpha$ and this pair of parameters could not in general, be associated with the experimental system unless the application is to a theory only weakly dependent on the potential, since the second virial coefficient associated with that function does not properly represent the low temperature second virial coefficient data. It is clear from figure 6 , therefore, that one must take the best fit of experimental data to a series of two parameter potentials (here the family of functions generated by varying the third parameter, $\alpha$ ) before assigning a particular potential to the experimental system. Unfortunately, the literature is full of fits of data to single potential functions, particularly to the $(12,6)$ potential. Quite often the resulting potential has been used as the intermolecular potential functions for particular systems in evaluations of theories [15]. According to our results one must be suspicious of conclusions as to the relation between the particular theory and experiment, based on such work, unless the theory is known to depend only weakly on the potential function.

\section{Zero Density Adiabatic Joule-Thomson Coefficient}

This quantity is obtained directly as the zero density limit of experimental free expansion data. To obtain the second virial coefficient, on the other hand, one must first, in some manner, differentiate the experimental P-V-T data with respect to the density and then take the zero density limit. As a result, given the same experimental precision, one obtains the latter with much less precision than the former. This is not a real advantage for the free expansion data at the present time, however, since such data can be obtained only with a precision orders of magnitude below that possible in P-V-T work, particularly at low densities.

The adiabatic Joule-Thomson coefficient depends both on the second virial coefficient and its first derivative. Clearly, where two second virial coefficients, one for each of two potentials, are indistinguishable over an extended temperature range, their first derivatives are also indistinguishable, at least for temperatures near the center of this range. Furthermore, this will be true for exactly the same parameter ratios. Differences which occur for the second virial coefficients near the edge of this temperature range necessarily appear as larger differences in their derivatives. Thus, one expects the range of equivalence for two potentials to be smaller for the zero density Joule-Thomson coefficient than it is for the second 
virial coefficient. Results for this quantity are contained in figures 7 and 8 . As expected, the range of equivalence of the potentials is shorter. It should be remembered that, since our method involves equating a property and its first derivative, equivalence here includes the second derivative of the second virial coefficient.

\section{Zero Density Viscosity and Diffusion Coefficients}

The $\epsilon_{2} / \epsilon_{1}$ ratios for the zero density viscosity and diffusion coefficients are contained in figures $6,7,9$, and 10 . The ratios $\left(b_{0}\right)_{2} /\left(b_{0}\right)_{1}$ for several potentials are presented in figure 11. In figure 7, for the Kihara potential, there is no curve for either of these properties which approaches that of the second virial coefficient in flatness. The ratio $\epsilon_{2} / \epsilon_{1}$, can be called independent of $T^{*}$ only in a very narrow temperature region about the maximum of the curve. Figure 9 (which is drawn to a different scale) does indicate an increasing degree of flatness with increasing $\gamma$.

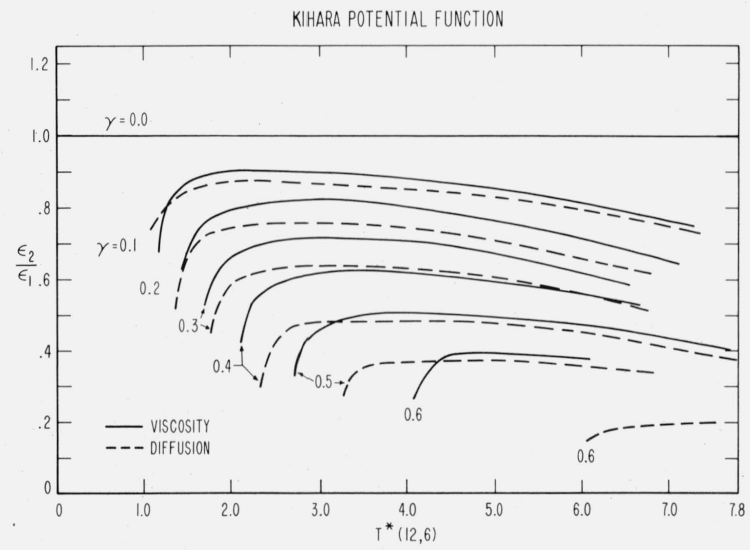

FIGURE 9. The parameter ratios $\epsilon_{2} / \epsilon_{1}$ for the diffusion and vis cosity coefficients for the Kihara potential function with respect to the $(12,6)$.

Notice the increasing flatness with increasing $\gamma$ for $T^{*}$ large.

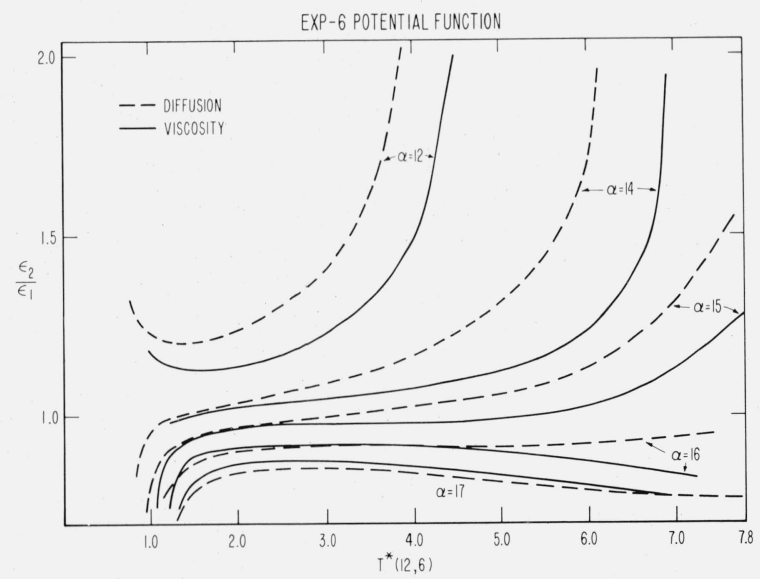

FIGURE 10. The parameter ratios $\boldsymbol{\epsilon}_{2} / \boldsymbol{\epsilon}_{1}$ for the diffusion and viscosity coefficients for the exp-6 potential with respect to the $(12,6)$ potential.

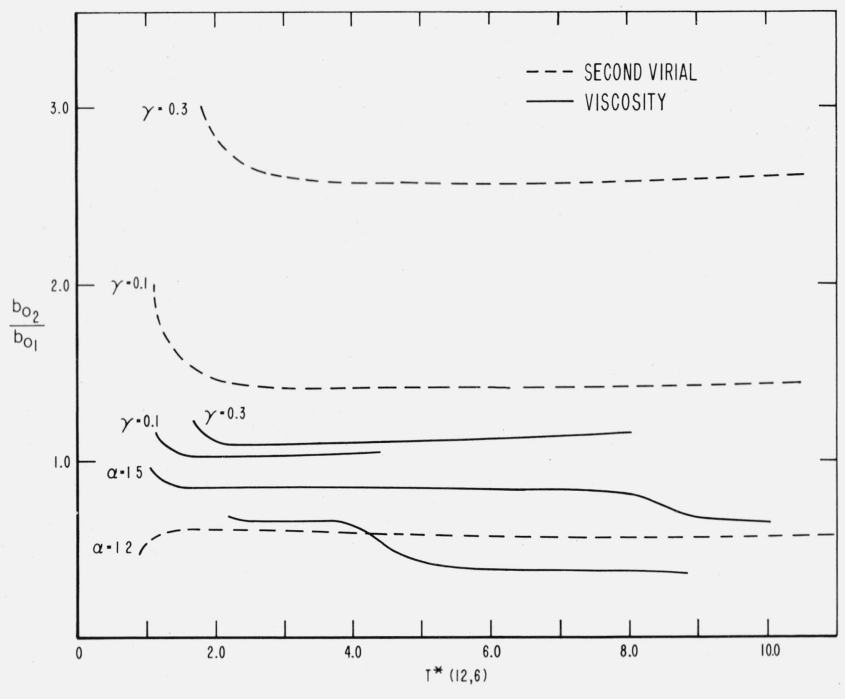

FIGURE 11. The parameter ratios $\left(\mathrm{b}_{0}\right)_{2} /\left(\mathrm{b}_{0}\right)_{1}$ for the Kihara and exp-6 potentials with respect to the $(12,6)$.

This occurs for relatively high temperature, $T>T_{B}$, however. This demonstrates the strong role played by the bowl of the potential function in determining the transport properties. For any given potential function, there is a temperature above which the transport properties are entirely dependent on the repulsive part of the potential function. Now, for the Kihara potential function, the position of the hard sphere cutoff moves to larger values of $r$ as $\gamma$ increases. Therefore, the temperature at which the properties for the Kihara potential become those for a hard sphere should decrease with increasing $\gamma$. According to figure 9, this is compensated for by the changes produced by the modification of the bowl with increasing $\gamma$. The net result is a set of properties (e.g., for $\gamma=0.6$ ) more nearly like those for the $\gamma=0$, (i.e., $(12,6))$ potential at the highest temperatures of figure 9. Further proof of the strong effect produced by the bowl can be seen in the behavior at low temperatures. At such temperatures, the repulsive part has a very minor effect on the properties. Therefore, the rapid variation in the dependence of $\epsilon_{2} / \epsilon_{1}$, with $T^{*}$ as a function of $\gamma$ at low temperatures is an indication of the marked effect of the bowl on these properties.

The curves in figure 10 are most flat for $T^{*}$ approximately equal to 2.0. It is interesting to note that there is a value of $\alpha$ for which the curve is quite flat at low temperatures as well. This occurs for $\alpha$ slightly larger than 13 for both properties. It should be noted that the corresponding ratio is very close to unity. Furthermore, for essentially the same value of $\alpha$, a flat curve with essentially the same ordinate obtained down to low temperatures for the second virial coefficient. Note that, unlike the case of the second virial coefficient, for these properties the 
curve for that value of $\alpha$ is not flat to very high temperatures. For these properties, a significant departure from unity occurs at $T^{*}=5.0$, not an impossibly high temperature for many systems. There is a tendency for the curves to flatten out at high temperatures for a value of $\alpha$ larger than 15 under which conditions the curves show a marked deviation from flatness at quite low temperatures. This behavior shows that it may be possible to distinguish between the $(12,6)$ potential and all members of the family of exp-6 functions given experimental transport data of sufficient accuracy which includes both the temperature ranges $T^{*}<2.0$ and $T^{*}>5.0$ on the $(12,6)$ scale. For argon this requires that there be data for $T<240{ }^{\circ} \mathrm{K}$ and $T>600{ }^{\circ} \mathrm{K}$ while for xenon $T<450{ }^{\circ} \mathrm{K}$ and $T>1225^{\circ} \mathrm{K}$. These conditions are met for argon but not for xenon, at the present time.

It is clear from these results that the diffusion and viscosity coefficients are potentially more sensitive probes of the potential function than either the second virial or Joule-Thomson coefficients given experimental data covering a sufficiently large temperature range. One might not have expected this since the latter properties are more simply related to the potential function than are the former. One might have expected a quantity like the second virial coefficient which is related to the potential function through a single integration to be much more sensitive to differences in that function than one like the transport properties which are connected by three integrations, particularly when the functional dependence in the integrand is also more indirect for the latter than it is for the former.

It must be remembered that the requirement here has been that both the value and slope of the properties be equal for the two potentials. It is possible that some of the apparent sensitivity found for the transport properties comes from the requirement on the slope. Whether or not this disappears when one asks only for a match to the property, as is done in a correlation, bears further study. Again one can state that a lack of uniqueness found in this calculation will not disappear on the application to experimental data. Therefore, one must have data present in the temperature ranges mentioned if one is even to have a chance to discriminate between potential functions.

The slope of the $\epsilon_{2} / \epsilon_{1}$ versus $T^{*}$ curve, for a given potential, is essentially the same for both the viscosity and diffusion coefficients. On this basis, the two quantities are equally effective when used individually. As we shall see in the next section, however, there is a difference between them in sensitivity to changes in the potential function. That difference makes the simultaneous fit of the two properties potentially a sensitive tool for finding the potential function.

The ratios $\left(b_{0}\right)_{2} /\left(b_{0}\right)_{1}$ for these properties are less dependent on temperature than are the ratios $\epsilon_{2} / \epsilon_{1}$. A discussion of them would therefore not contribute anything new with regard to the sensitivity of these properties as probes of the potential function.

\section{The Simultaneous Fit to More Than One Property}

Figures 6, 7, 9, and 10 contain plots of the ratio $\epsilon_{2} / \epsilon_{1}$ for more than one property. With the help of these one can examine the effect the requirement of a simultaneous fit to two or more properties and their first derivatives has on the lack of uniqueness in the potential function. Figure 9 is particularly informative in this regard. Note that in that diagram, the curves for both the viscosity and diffusion coefficients for $\gamma=0$ would be straight lines parallel to the abscissa at $\epsilon_{2} / \epsilon_{1}=1.0$. As $\gamma$ deviates from zero, however, each curve begins to deviate from a straight line, particularly at low temperatures. Furthermore, the curves for the diffusion coefficient separate from those for the viscosity until, by $\gamma=0.6$, the curves are quite widely separated. The lack of uniqueness associated with the flatness of the curves for the separate properties would be extended to the simultaneous fit to the pair of properties and their derivatives were the two curves essentially flat at the same value of the ordinate. However, the increase in the separation of the curves with $\gamma$ (i.e., with increased deviation from the $(12,6)$ reference potential) demonstrates a strong sensitivity of the simultaneous fit to changes in the potential function. This sensitivity is much stronger than that of the individual properties in the range $T^{*}>4.0$. Whether or not it is more sensitive than the behavior for $T^{*}<4.0$ would require an examination of detailed fits to data.

Figure 10 contains the same kind of information for the exp-6 function. Here the changes that occur at low and intermediate temperatures are much smaller while those at high temperatures are much more pronounced. There appears to be a value of $\alpha$ for which the curves both have a flat range and are essentially superimposed at high temperatures. However, for that value of $\alpha$, the low temperature data still serves as a discriminant. In this case the simultaneous use of both properties and their derivatives does not appear to add any discrimination beyond that available with either property by itself. Note that the curves for the two properties exchange relative positions when one goes from $\alpha=12$ to $\alpha=17$. Increasing $\alpha$ beyond 17 would presumably result in further separation.

Figure 7 contains plots for all of the properties for the Kihara potential. These curves necessarily each form a straight line parallel to the abscissa with ordinate 1.0 for $\gamma=0$. Increasing $\gamma$ causes them to separate as in figure 9 for the two transport properties. It is interesting to note that the change in separation between the curves for diffusion and second virial coefficients at intermediate temperatures is not uniform. Thus, for $T^{*} \cong 3.0$, the diffusion curve for $\gamma=0.1$ lies above that for the second virial coefficient, for $\gamma=0.3$ they are superimposed in a small region around $T^{*}=3.0$, while for $\gamma=0.4$ the diffusion curve lies below. Thus, as $\gamma$ is increased from 0 . to 0.1 , the second virial curve "moves" more rapidly while for $\gamma>0.1$ the reverse is true. 


\section{Conclusions}

We have investigated the use of the second virial coefficient as well as the zero density Joule-Thomson, viscosity, and diffusion coefficients as probes for the intermolecular potential function. We have found the second virial coefficient to be particularly poor in this regard in the temperature range $2.0<T^{*}<7.0$ on the Lennard-Jones $(12,6)$ reduced temperature scale. The Joule-Thomson coefficient has been found to be somewhat better particularly in the lower part of this range. Considerably better were the transport coefficients, i.e., the viscosity and diffusion coefficients. It is clear from our results that these last coefficients can be sensitive probes of the potential function given experimental data covering a sufficiently large temperature range.

Further comparisons of this kind between the transport coefficients for the $(12,6)$ potential function and those for other potential functions are clearly called for, particularly for classes of potentials of which the $(12,6)$ is not a member. In particular, one would like to find such classes of functions as exist which contain a member whose transport properties can be fit to those of the $(12,6)$ over an extensive temperature range. This will require the calculation of collision integrals for these various functions. In some cases tables do exist $[16,17]$ which, unfortunately, contain too few points in the temperature region of interest. It is hoped that these will be extended shortly.

These calculations will be extended to include the thermal diffusion ratio and, possibly, the quantum corrections to certain of these properties.

As regards the relationship between our results and experiment with present day precision, the fact that our results apply precisely to experiment only in the limit of very high precision actually strengthens our conclusions in regard to lack of uniqueness while conclusions regarding uniqueness are weakened. That is, where we did not find it possible to use a macroscopic property to distinguish between potential functions under our conditions, it certainly would not be possible to use this property for this purpose under less precise experimental conditions. On the other hand, our ability in other circumstances to distinguish among potentials (e.g., using second virial coefficient data for $T^{*}(12,6,<2.0)$ may be due in part, to the fact that we have required a precise fit, something not possible with experimental data. It may likewise be due to the inclusion here of a requirement that the first derivative of the property be equal for the two potentials. More precise statements in this latter case await the result of fits to actual data.

Early portions of this work were completed at the Weizmann Institute, Rehovoth, Israel. The author wishes particularly to thank the computer staff for their cooperation in the use of their CDC-1604 computer.

\section{References and Notes}

[1] M. Klein, J. Chem. Phys. 39, 1367 (1963).

[2] J. Hilsenrath and M. Klein, Tables of Thermodynamic Properties of Air from $1500^{\circ} \mathrm{K}$ to $15000^{\circ} \mathrm{K}$, Report AEDC TR65-58 Arnold Engineering Development Center, Tullahoma, Tenn.

[3] J. O. Hirschfelder, C. F. Curtiss, and R. B. Bird, The Molecular Theory of Gases and Liquids (John Wiley \& Sons, Inc., New York, N.Y., 1954).

[4] J. B. Keller, and B. Zumino, J. Chem. Phys. 30, (1959); J. E. Le Fevre, Heat Division Paper 129 (1957), Mechanical Engineering Research Laboratory, East Kilbride, Glasgow, Scotland.

[5] Note that equating the second virial coefficients and their first derivatives is equivalent to equating, for the two potentials, the first density correction to PV/RT and simultaneously requiring the first density corrections to the internal energy, U/RT, to be equal for the same two potentials [6]. The author is indebted to Lester Haar for pointing this out.

[6] The requirement on the internal energy can be replaced by an equivalent requirement on the zero density Joule-Thomson coefficient.

[7] One can also associate each curve of the family of curves with $T_{2}^{*}$ or, for that matter, with the experimental temperature $T$.

[8] M. Klein, Ph.D. thesis, 1962, University of Maryland.

[9] Note that $S$ is dependent on $T^{*}$ and contrary to the statement in J. O. Hirschfelder, C. F. Curtiss, and E. M. Spotz, Chem. Rev. 44, 205 (1949), (see also p. 566 ref. 3) is not the exponent in the representation $B\left(T^{*}\right)=A T^{* t}$.

[10] e.g., R. A. Svehla, NASA-TRR-132 (1962); see also reference 2.

[11] C. J. G. Raw, J. South African Chemical Institute VII, 96 (1954).

[12] As regards the second virial coefficient and hence low density P.V-T data, this weakens considerably one of the restrictions of the law of corresponding states in this temperature range. Thus, at these temperatures, one needs to place only mild restrictions on the form of the potential function.

[13] E. Whalley and W. G. Schneider, J. Chem. Phys. 23, 1644 (1955).

[14] E. A. Mason and W. E. Rice, Jr., J. Chem. Phys. 22, 843 (1954).

[15] e.g., L. Verlet, and D. Levesque, Physica 38, 1124 (1962); J. deBoer, J. M. H. van Leeuwen, and J. Groenevelt, Physica 30, 2265 (1964); J. G. Kirkwood, V. A. Lewison, and B. J. Alder, J. Chem. Phys. 20, 929 (1952); G. Casanova, and A. Levi, Physica 30, 937 (1964).

[16] F. J. Smith and R. J. Munn, J. Chem. Phys. 41, 3560 (1964).

[17] S. G. Brush and J. D. Lawrence, Report UCRL-7376 Lawrence Radiation Laboratory, Livermore, Calif.

(Paper 70A3-402) 